\title{
Asymptotic behavior of a stochastic HIV model with Beddington-DeAngelis functional response
}

\author{
Suxia Wang ${ }^{1,2}$, Juan Zhao ${ }^{{ }^{*}}$, Junxing Zhu ${ }^{1}$ and Xiaoli Ren ${ }^{1,2}$
}

\section{"Correspondence:}

zhaojuan@nudt.edu.cn

${ }^{1}$ College of Computer Meteorology and Oceanography, National

University of Defense Technology, Changsha, China

Full list of author information is available at the end of the article

\begin{abstract}
In this paper, we study the dynamics property of a stochastic HIV model with Beddington-DeAngelis functional response. It has a unique uninfected steady state. We prove that the model has a unique global positive solution. Furthermore, if the basic reproductive number is not larger than 1, the asymptotic behavior of the solution is stochastically stable. Otherwise, it fluctuates randomly around the infected steady state of the corresponding deterministic HIV model. Finally, some numerical simulations are carried out to verify our results.
\end{abstract}

Keywords: Stochastic HIV model; Itô's formula; Asymptotic behavior; Lyapunov function; Beddington-DeAngelis functional

\section{Introduction}

Acquired Immune Deficiency Syndrome (AIDS) has been one of the most dangerous epidemic diseases in the world since its discovery in 1981. It is caused by the HIV virus, which invades the $\mathrm{CD}^{+} \mathrm{T}$ cells of the human body, depletes them, and leads to the destruction of the immune system. To block the spread of AIDS, HIV viral dynamics urgently requires study. Abundant research of the dynamic characteristics of the HIV virus has played an important role in preventing the spread of AIDS [1-3].

Hernandez-Vargas and Middleton [1] noted that a typical HIV infection response has three stages: an initial acute infection, a long asymptomatic period, and a final increase in viral load with simultaneous collapse in healthy $\mathrm{CD} 4^{+} \mathrm{T}$ cell counts. A deterministic model was proposed to explain the three stages of the infection, including the progression of AIDS. Nowak et al. [2] established mathematical models based on HIV infection responses, which can provide nonintuitive insights into the dynamics of host responses to infectious agents and new avenues for experimentation. Motivated by the research of Nowak et al., Huang et al. [3] investigated the global stability of a deterministic virus dynamics model with a Beddington-DeAngelis infection rate which is given by

$$
\left\{\begin{array}{l}
\dot{x}=\lambda-q x-\frac{\beta x v}{1+a x+b v}, \\
\dot{y}=\frac{\beta x v}{1+a x+b v}-p y, \\
\dot{v}=k y-u v .
\end{array}\right.
$$

(c) The Author(s) 2020. This article is licensed under a Creative Commons Attribution 4.0 International License, which permits use, sharing, adaptation, distribution and reproduction in any medium or format, as long as you give appropriate credit to the original author(s) and the source, provide a link to the Creative Commons licence, and indicate if changes were made. The images or other third party material in this article are included in the article's Creative Commons licence, unless indicated otherwise in a credit line to the material. If material is not included in the article's Creative Commons licence and your intended use is not permitted by statutory regulation or exceeds the permitted use, you will need to obtain permission directly from the copyright holder. To view a copy of this licence, visit http://creativecommons.org/licenses/by/4.0/. 
Here $x(t), y(t)$, and $v(t)$ denote the concentration of uninfected $\mathrm{CD}^{+}{ }^{+} \mathrm{T}$ cells, the concentration of infected $C D 4^{+} \mathrm{T}$ cells that produce the virus, and the concentration of HIV virus at time $t$, respectively. The parameters in the model are interpreted as follows:

$\lambda$ : the growth rate of uninfected $\mathrm{CD} 4^{+} \mathrm{T}$ cells,

$\beta$ : the transmission coefficient between uninfected cells and infected virus particles,

$q$ : the death rate of uninfected $\mathrm{CD} 4^{+} \mathrm{T}$ cells,

$p$ : the death rate of infected $\mathrm{CD} 4^{+} \mathrm{T}$ cells,

$u$ : the rate at which virus particles are removed from the model,

$k$ : the rate at which new viruses are produced from the division of the infected cells. All the parameters above are nonnegative. $\frac{\beta x v}{1+a x+b v}$ is the Beddington-DeAngelis functional response of the infection rate of HIV, where $a, b \geq 0$ are constants.

The Beddington-DeAngelis functional response $[4,5]$ can reflect that mutual interference between HIV viruses and the concentration of uninfected $\mathrm{CD}^{+} \mathrm{T}$ cells influences the infection rate. It can describe nonlinear incidence rate including bilinear functional response [6, 7], Holling type II functional response [8] and saturation response [9]. So it can provide a better description of the process showing how HIV infects healthy $\mathrm{CD} 4^{+} \mathrm{T}$ cells, and the model is more realistic than most models.

The basic reproductive ratio of the virus for the model (1.1) is $R_{0}=\frac{\lambda \beta k}{p q u+a p u \lambda}$ in [3]. If $R_{0} \leq 1$, the model (1.1) has a unique uninfected steady state $E_{0}=\left(\frac{\lambda}{q}, 0,0\right)$. If $R_{0}>1$, there exists an infected steady state $E^{*}=\left(x^{*}, y^{*}, v^{*}\right)$ which is given by

$$
\begin{aligned}
x^{*} & =\frac{\lambda b k+p u}{k \beta+b k q-a p u}, \\
y^{*} & =\frac{\lambda \beta k}{p(k \beta+b k q-a p u)}\left(1-\frac{1}{R_{0}}\right), \\
v^{*} & =\frac{\lambda \beta k^{2}}{p u(k \beta+b k q-a p u)}\left(1-\frac{1}{R_{0}}\right) .
\end{aligned}
$$

The steady states are always globally asymptotically stable for the model (1.1).

Yan et al. [10] proposed a mathematical model injected with a combination of HIV latency activators to reduce persistent HIV reservoirs, and they achieved the optimal time for cessation of treatment by combining the basic reproductive ratio of virus and therapy of inducers and antibodies.

In fact, the transmission process of epidemic diseases is often affected by random factors. In a complex and changing environment, biochemical reactions have randomness. The randomness is introduced into the process of infection of $\mathrm{CD}^{+} \mathrm{T}$ cells and the transitions from uninfected to latently or actively infected cells by chance mechanisms in the early period of infection. Using the stochastic analyses can provide a more accurate quantitative basis than deterministic in real systems [11]. And the effective population size may be rather small in the initial stage of infection. Under the present circumstances, the HIV model is best described by a stochastic evolutionary model. Deterministic models are only appropriate when population sizes are large $[12,13]$.

The stochasticity is often described by white noise $\xi(\cdot)$ [14-16]. In the early work, asymptotic behavior of the solution of stochastic HIV virus models with white noise has been studied and compared to the corresponding deterministic model. The dynamic behavior of these HIV models provides a strong theoretical basis for the prevention and treat- 
ment of AIDS. Dalal et al. [6] built a stochastic model representing HIV internal virus dynamics in which the death rate of uninfected $\mathrm{CD} 4^{+} \mathrm{T}$ cells is disturbed by white noise, and they analyzed the asymptotic behavior of the model. Wang et al. [17] gave the asymptotic dynamics of the model depend on the general incidence function and noise intensities. Liu et al. [18] claimed a stochastic predator-prey system with Beddington-DeAngelis functional response, which can provide better descriptions of predator feeding over a range of predator-prey abundances. But the asymptotic dynamics of the stochastic model was governed by a threshold parameter. So we concentrate on the solution in a HIV stochastic system there being a Beddington-DeAngelis functional response or not and see what dynamic behavior there is, and whether the solution is affected by white noise. The existing HIV stochastic models can be improved and generalized under certain assumptions.

In our paper, we introduce randomness to a model based on the HIV deterministic virus model (1.1) with Beddington-DeAngelis functional response, by replacing the parameter $\beta$ by $\beta+\sigma \dot{B}(t)$, where $B(t)$ is the standard Brownian motion and $\sigma^{2} \geq 0$ represents the intensity of $B(t)$. We establish this stochastic HIV model as

$$
\left\{\begin{array}{l}
d x=\left(\lambda-q x-\frac{\beta x v}{1+a x+b v}\right) d t-\sigma \frac{x v}{1+a x+b v} d B(t) \\
d y=\left(\frac{\beta x v}{1+a x+b v}-p y\right) d t+\sigma \frac{x v}{1+a x+b v} d B(t) \\
d v=(k y-u v) d t
\end{array}\right.
$$

Obviously, if $R_{0} \leq 1, E_{0}$ still is in the uninfected steady state in the model (1.1), but when $R_{0}>1$, the infected steady state $E^{*}$ is not a steady state for the model (1.2). However, if $R_{0}>1$, we can discuss the asymptotic behavior of the solution of the model (1.2) around the infected steady state $E^{*}$ of the model (1.1). We use a mathematical method based on previous work $[7,19]$.

The rest of this paper is organized as follows. In Sect. 2, we prove the positivity of the solution in model (1.2). We study whether the uninfected steady state of the model (1.2) is stochastically stable in Sect. 3. In Sect. 4, we discuss the asymptotic behavior of the global positive solution in model (1.2) oscillating around the infected steady state of the model (1.1) under certain parametric conditions. We present some numerical simulations in Sect. 5, and conclusions are offered in Sect. 6.

\section{Global positive solution}

Unless otherwise specified, we let $\left(\Omega, \mathcal{F},\left\{\mathcal{F}_{t}\right\}_{t \geq 0}, \mathbb{P}\right)$ be a complete probability space with a filtration $\left\{\mathcal{F}_{t}\right\}_{t \geq 0}$ satisfying the usual conditions (i.e., it is increasing and right continuous while $\mathcal{F}_{0}$ contains all $\mathbb{P}$-null sets). $B(t)$ is the standard Brownian motion defined on this probability space. Moreover, let $R_{+}^{3}=\left\{(x(t), y(t), v(t)) \in R^{3}: x(t), y(t), v(t)>0\right\}$.

We address the $\mathrm{n}$-dimensional stochastic differential equation [16]

$$
d x(t)=f(x(t), t) d t+g(x(t), t) d B(t), \quad t \geq t_{0} .
$$

Assuming that $C^{2,1}\left(S_{h} \times\left[t_{0},+\infty\right) ; R_{+}\right)$is the family of all nonnegative functions $V(x, t)$ defined on $\left(\left[t_{0},+\infty\right) ; R_{+}\right)$, such that they are continuously twice differentiable in $x$ and once in $t$, then

$$
d V(x(t), t)=L V(x(t), t) d t+V_{x}(x(t), t) g(t) d B(t),
$$


where

$$
L V(x(t), t)=V_{t}(x(t), t)+V_{x}(x(t), t) f(t)+\frac{1}{2} \operatorname{tr}\left[g^{T}(t) V_{x x}(x(t), t) g(t)\right], \quad \text { a.s. }
$$

Theorem 2.1 For any initial value $(x(0), y(0), v(0)) \in R_{+}^{3}$, there is a unique solution $(x(t), y(t), v(t))$ of the model (1.2) on $t \geq 0$, and the solution will remain in $R_{+}^{3}$ with probability 1.

Proof Since the coefficients of the equation are locally Lipschitz continuous, for any given initial value $(x(0), y(0), v(0)) \in R_{+}^{3}$, there is a unique local solution $(x(t), y(t), v(t))$ on $t \in$ $\left[0, \tau_{e}\right)$, where $\tau_{e}$ is the explosion time $[20,21]$.

Setting $N(t)=x(t)+y(t)$, we obtain from the model (1.2)

$$
d N(t)=(\lambda-q x-p y) d t
$$

i.e.,

$$
\frac{d N(t)}{d t}=\lambda-q x-p y
$$

Because $x(t) \geq 0$ and $y(t) \geq 0$ on $t \in\left[0, \tau_{e}\right)$, we have

$$
\lambda-\max \{p, q\} N(t) \leq \frac{d N(t)}{d t} \leq \lambda-\min \{p, q\} N(t) .
$$

$N_{1}(t)$ is the solution of the equation for any initial value $(x(0), y(0), v(0)) \in R_{+}^{3}$ :

$$
\left\{\begin{array}{l}
d N_{1}(t)=\left(\lambda-\min \{p, q\} N_{1}(t)\right) d t, \\
N_{1}(0)=N(0),
\end{array}\right.
$$

and we get

$$
N_{1}(t)=\frac{\lambda}{\min \{p, q\}}+\left(N(0)-\frac{\lambda}{\min \{p, q\}}\right) \exp (-\min \{p, q\} t) .
$$

By the differential equation comparison theorem $[19,22]$, we get

$$
N(t) \leq N_{1}(t) \leq \max \left\{\frac{\lambda}{\min \{p, q\}}, N(0)\right\}:=N_{\max }
$$

and

$$
x(t), y(t) \leq N_{\max }, \quad t \in\left[0, \tau_{e}\right) \text { a.s. }
$$

Simultaneously,

$$
N(t) \geq \min \left\{\frac{\lambda}{\max \{p, q\}}, N(0)\right\}:=N_{\min }
$$


and

$$
x(t), y(t) \geq N_{\min }, \quad t \in\left[0, \tau_{e}\right) \text { a.s. }
$$

For

$$
\frac{d v(t)}{d t}=k y-u v \leq k N_{\max }-u v,
$$

simultaneously, we obtain

$$
v(t) \leq \max \left\{\frac{k N_{\max }}{u}, v(0)\right\}:=V_{\max }, \quad t \in\left[0, \tau_{e}\right) \text { a.s. }
$$

To show that this solution is global, we must have $\tau_{e}=\infty$ a.s. Set $k_{0}>0$ to be sufficiently large so that each component of $(x(0), y(0), v(0))$ is in $\left[1 / k_{0}, k_{0}\right]$. For each integer $k \geq k_{0}$, define the stopping time as

$$
\tau_{k}=\inf \left\{t \in\left[0, \tau_{e}\right): x(t) \notin\left(\frac{1}{k}, k\right) \text { or } y(t) \notin\left(\frac{1}{k}, k\right) \text { or } v(t) \notin\left(\frac{1}{k}, k\right)\right\} \text {, }
$$

where we set $\inf \emptyset=\infty$ ( $\varnothing$ denotes the empty set).

Evidently, $\tau_{k}$ is monotonically increasing as $k \rightarrow \infty$. Set $\tau_{\infty}=\lim _{k \rightarrow \infty} \tau_{k}$, then $\tau_{\infty} \leq \tau_{e}$ a.s. If we can prove that $\tau_{\infty}=\infty$ a.s., then $\tau_{e}=\infty$ and $(x(t), y(t), v(t)) \in R_{+}^{3}$ a.s. are obtained for $t \geq 0$. That is, we are just to prove $\tau_{\infty}=\infty$. Assuming $\tau_{\infty} \neq \infty$ a.s., there exist constants $T>0$ and $\epsilon \in(0,1)$ such that $P\left\{\tau_{\infty}\right\} \leq T>\epsilon$.

So, there is an integer $k_{1} \geq k_{0}$, and

$$
P\left\{\tau_{k} \leq T\right\} \geq \epsilon, \quad \forall k \geq k_{1}
$$

Define a positive definite Lyapunov function:

$$
V(x, y, v)=x-1-\ln (x)+y-1-\ln (y)+v-1-\ln (v) .
$$

If $t \in\left[0, \tau_{k}\right)$, then $x(t), v(t), v(t) \in[1 / k, k]$. The non-positivity of this function can be seen from $u+1-\ln (u) \geq 0$ for $u>0$ and Itô's formula [16, 20, 21], and we get

$$
d V(x, y, v)=L V(x, y, v) d t+\left(\frac{1}{x}-\frac{1}{y}\right) \frac{\sigma x v}{1+a x+b v} d B(t)
$$

where

$$
\begin{aligned}
L V(x, y, v)= & \left(1-\frac{1}{x}\right)\left(\lambda-q x-\frac{\beta x v}{1+a x+b v}\right)+\left(1-\frac{1}{y}\right)\left(\frac{\beta x v}{1+a x+b v}-p y\right) \\
& +\left(1-\frac{1}{v}\right)(k y-u v)+\frac{1}{2} \frac{1}{x^{2}}\left(-\frac{\sigma x v}{1+a x+b v}\right)^{2}+\frac{1}{2} \frac{1}{y^{2}}\left(\frac{\sigma x v}{1+a x+b v}\right)^{2} \\
\leq & \lambda+q+p+u+k y+\frac{\beta v}{1+a x+b v}+\frac{\sigma^{2} v^{2}}{2(1+a x+b v)^{2}}+\frac{\sigma^{2} x^{2} v^{2}}{2 y^{2}(1+a x+b v)^{2}} \\
\leq & \lambda+q+p+u+k y+\beta v+\frac{1}{2} \sigma^{2} v^{2}+\frac{1}{2} \frac{\sigma^{2} x^{2} v^{2}}{y^{2}}
\end{aligned}
$$




$$
\begin{aligned}
& \leq \lambda+q+p+u+k N_{\max }+\beta V_{\max }+\frac{1}{2} \sigma^{2} V_{\max }^{2}+\frac{1}{2} \frac{\sigma^{2} N_{\max }^{2} V_{\max }^{2}}{N_{\min }^{2}} \\
& :=F .
\end{aligned}
$$

So, we obtain

$$
d V(x, y, v) \leq F d t+G d B(t)
$$

where $G=\left(\frac{1}{x}-\frac{1}{y}\right) \frac{\sigma x v}{1+a x+b v}$.

$$
\text { If } 0<t_{1}<T \text {, then }
$$

$$
\int_{0}^{\tau_{k} \wedge t_{1}} d V(x(t), y(t), v(t)) \leq \int_{0}^{\tau_{k} \wedge t_{1}} F d t+\int_{0}^{\tau_{k} \wedge t_{1}} G d B(t) .
$$

Therefore,

$$
\begin{aligned}
& E\left(V\left(x\left(\tau_{k} \wedge t_{1}\right), y\left(\tau_{k} \wedge t_{1}\right), v\left(\tau_{k} \wedge t_{1}\right)\right)\right) \\
& \quad \leq V(x(0), y(0), v(0))+E \int_{0}^{\tau_{k} \wedge t_{1}} F d t \\
& \quad=V(x(0), y(0), v(0))+F E\left(\tau_{k} \wedge t_{1}\right) \leq V(x(0), y(0), v(0))+F T .
\end{aligned}
$$

Set $\Omega_{k}=\left\{\tau_{k} \leq T\right\}$ for $k \geq k_{1}$. Then, by (2.2), $P\left(\Omega_{k}\right) \geq \epsilon$. Note that, for every $\omega \in \Omega_{k}$, there is $x\left(\tau_{k}, \omega\right), y\left(\tau_{k}, \omega\right)$ or $v\left(\tau_{k}, \omega\right)$ that equals either $k$ or $1 / k . V\left(x\left(\tau_{k}, \omega\right), y\left(\tau_{k}, \omega\right), v\left(\tau_{k}, \omega\right)\right)$ is no smaller than either

$$
k+1-\ln (k) \quad \text { or } \quad 1 / k+1-\ln (1 / k)=1 / k+1+\ln (k) .
$$

Hence

$$
V\left(x\left(\tau_{k}, \omega\right), y\left(\tau_{k}, \omega\right), v\left(\tau_{k}, \omega\right)\right) \geq[k+1-\ln (k)] \wedge[1 / k+1+\ln (k)] .
$$

It follows from (2.3) that

$$
\begin{aligned}
V(x(0), y(0), v(0))+F T & \geq E\left[1_{\Omega_{k}} V\left(x\left(\tau_{k}, \omega\right), y\left(\tau_{k}, \omega\right), v\left(\tau_{k}, \omega\right)\right)\right] \\
& \geq \epsilon([k+1-\ln (k)] \wedge[1 / k+1+\ln (k)]),
\end{aligned}
$$

where $1_{\Omega_{k}}$ is the indicator function of $\Omega_{k}$. Letting $k \rightarrow \infty$, we obtain $V(x(0), y(0), v(0))+$ $F T \geq \infty$, leading to the contradiction with $V(x(0), y(0), v(0))+F T<\infty$. So, we must have $\tau_{\infty}=\infty$ a.s.

Remark 2.2 For any initial value $(x(0), y(0), v(0)) \in R_{+}^{3}$, we have $N_{\min } \leq x(t)+y(t) \leq N_{\max }$, $v(t) \leq V_{\max }$ a.s. for the model (1.2) on $t \geq 0$, where

$$
\begin{aligned}
& N_{\max }=\max \left\{\frac{\lambda}{\min \{p, q\}}, N(0)\right\}, \quad N_{\min }=\min \left\{\frac{\lambda}{\max \{p, q\}}, N(0)\right\}, \\
& N(0)=x(0)+y(0), \quad V_{\max }=\max \left\{\frac{k N_{\max }}{u}, v(0)\right\} .
\end{aligned}
$$




\section{Stochastic stability of uninfected steady state for the stochastic HIV model}

If $R_{0} \leq 1$, there is a globally asymptotically stable uninfected steady state $E_{0}=\left(\frac{\lambda}{q}, 0,0\right)$ in deterministic model (1.1). Obviously, $E_{0}$ is also an uninfected steady state of the stochastic HIV model (1.2). We now prove the stochastic asymptotic stability of the uninfected steady state $E_{0}$.

Lemma 3.1 ([16]) There exists a positive definite function $V(x, t) \in C^{2,1}\left(S_{h} \times\left[t_{0},+\infty\right) ; R_{+}\right)$ such that $L V(x, t) \leq 0$, then the trivial solution of Eq. (2.1) is stochastically stable.

Theorem 3.2 Assuming that $R_{0} \leq 1$ and $\sigma^{2}<\frac{2 p u b}{k\left(1+a N_{\max }+b N_{\max }\right) x_{0}}$, then, for any given initial value $(x(0), y(0), z(0)) \in R_{+}$, the infection-free equilibrium $E_{0}$ for the model (1.2) is stochastically stable.

Proof Define a Lyapunov function,

$$
V_{1}(x, y, v)=\frac{x_{0}}{1+a x_{0}}\left(\frac{x}{x_{0}}-1-\ln \frac{x}{x_{0}}\right)+y+\frac{p}{k} v
$$

where $x_{0}=\frac{\lambda}{q}$, and $V_{1}$ is a positive definite function with respect to $\left(x-x_{0}, y, v\right)$. Utilizing Itô's formula, we compute

$$
d V_{1}(x, y, v)=L V_{1}(x, y, v) d t+\left[1-\frac{x_{0}}{1+a x_{0}}\left(\frac{1}{x_{0}}-\frac{1}{x}\right)\right] \frac{\sigma x v}{1+a x+b v} d B(t)
$$

In detail,

$$
\begin{aligned}
L V_{1}(u, v, z)= & \frac{x_{0}}{1+a x_{0}}\left(\frac{1}{x_{0}}-\frac{1}{x}\right)\left(\lambda-q x-\frac{\beta x v}{1+a x+b v}\right)+\frac{1}{2} \cdot \frac{x_{0}}{1+a x_{0}} \\
& \cdot \frac{1}{x^{2}} \cdot\left(-\frac{\sigma x v}{1+a x+b v}\right)^{2}+\frac{\beta x v}{1+a x+b v}-p y+\frac{p}{k}(k y-u v) \\
= & \frac{q x_{0}}{1+a x_{0}}\left(2-\frac{x}{x_{0}}-\frac{x_{0}}{x}\right)+\frac{\beta x v}{1+a x+b v}+\frac{1}{1+a x_{0}} \cdot \frac{\beta x_{0} v}{1+a x+b v} \\
& -\frac{1}{1+a x_{0}} \cdot \frac{\beta x v}{1+a x+b v}-\frac{p u}{k} v+\frac{\sigma^{2} x_{0} v^{2}}{2\left(1+a x_{0}\right)(1+a x+b v)^{2}} \\
\leq & \frac{q x_{0}}{1+a x_{0}}\left(2-\frac{x}{x_{0}}-\frac{x_{0}}{x}\right)+\frac{p u(1+a x) v}{k(1+a x+b v)}\left(R_{0}-1\right) \\
& -\left[\frac{p u b}{k(1+a x+b v)}-\frac{\sigma^{2} x_{0}}{2}\right] v^{2} \\
\leq & \frac{q x_{0}}{1+a x_{0}}\left(2-\frac{x}{x_{0}}-\frac{x_{0}}{x}\right)+\frac{p u(1+a x) v}{k(1+a x+b v)}\left(R_{0}-1\right) \\
& -\left[\frac{p u b}{k\left(1+a N_{\max }+b N_{\max }\right)}-\frac{\sigma^{2} x_{0}}{2}\right] v^{2} .
\end{aligned}
$$




$$
\begin{aligned}
& \text { If } \sigma^{2}<\frac{2 p u b}{k\left(1+a N_{\max }+b N_{\max }\right) x_{0}}, \text { then } \\
& \qquad \begin{aligned}
L V_{1}(x, y, v) \leq & \frac{q x_{0}}{1+a x_{0}}\left(2-\frac{x}{x_{0}}-\frac{x_{0}}{x}\right)+\frac{p u v(1+a x)}{k(1+a x+b v)}\left(R_{0}-1\right) \\
& -\left[\frac{p u b}{k\left(1+a N_{\max }+b N_{\max }\right)}-\frac{\sigma^{2} x_{0}}{2}\right] v^{2} \\
\leq & 0 .
\end{aligned}
\end{aligned}
$$

So, the infection-free equilibrium $E_{0}$ in model (1.2) is stochastically stable according to Lemma 3.1. The proof is completed.

\section{Asymptotic behavior around infected steady state of the deterministic model}

If $R_{0}>1$, the infected steady state $E^{*}=\left(x^{*}, y^{*}, v^{*}\right)$ of the deterministic model (1.1) is globally asymptotically stable. However, $E^{*}$ is not the infected steady state of the stochastic model (1.2). Hence, we discuss asymptotic behavior of the solution for the stochastic model (1.2) around $E^{*}=\left(x^{*}, y^{*}, v^{*}\right)$.

Theorem 4.1 Assuming that $R_{0}>1$ and

$$
\sigma^{2}<\min \left\{\frac{q}{N_{m}^{2} B}, \frac{A}{N_{m}^{2} B}\right\}
$$

the solution of the model (1.2) for any given initial value $(x(0), y(0), v(0)) \in R_{+}^{3}$ has the following properties:

$$
\limsup _{t \rightarrow \infty} \frac{1}{t} E \int_{0}^{t}\left[\left(x(r)-x^{*}\right)^{2}+\left(y(r)-y^{*}\right)^{2}+\left(v(r)-v^{*}\right)^{2}\right] d r \leq \frac{K}{M}
$$

where

$$
\begin{aligned}
& N_{m}=\max \left\{N_{\max }, V_{\max }\right\}, \quad c_{1}=\frac{N_{m}\left[1+(a+b) N_{m}\right](p+q)^{2}}{2 p q\left(1+b v^{*}\right)}, \\
& A=\frac{p u^{2}}{4 k^{2}}+\frac{c_{1} b p y^{*}}{v^{*}\left[1+(a+b) N_{m}\right]^{2}}, \quad B=c_{1}\left(\frac{1+b v^{*}}{\beta v^{*} x^{* 2}}+\frac{y^{*}}{N_{\min }^{2}}\right), \\
& K=\max \left\{B N_{2}^{2} \sigma^{2} x^{* 2}, B N_{2}^{2} \sigma^{2} v^{* 2}\right\}, \quad M=\min \left\{q-B N_{m} \sigma^{2}, A-B N_{m} \sigma^{2}, \frac{p}{4}\right\} .
\end{aligned}
$$

Proof From the model (1.1), we have

$$
\lambda=q x^{*}+p y^{*}, \quad \frac{p u}{k}=\frac{p y^{*}}{v^{*}}, \quad p y^{*}=\frac{\beta x^{*} v^{*}}{1+a x^{*}+b v^{*}} .
$$

Define a Lyapunov function:

$$
V_{2}(x, y, v)=V_{21}(x, y, v)+\frac{p u}{2 k^{2}} V_{22}(x, y, v)+c_{1} V_{23}(x, y, v)
$$


where $c_{1}$ is the positive constant given by Theorem 4.1, and

$$
\begin{aligned}
V_{21}(x, y, v)= & \frac{1}{2}\left(x-x^{*}+y-y^{*}\right)^{2}, \\
V_{22}(x, y, v)= & \frac{1}{2}\left(v-v^{*}\right)^{2}, \\
V_{23}(x, y, v)= & x-x^{*}-\int_{x^{*}}^{x} \frac{p y^{*}}{\frac{\beta \tau v^{*}}{1+a \tau+b v^{*}}} d \tau+y-y^{*}-y^{*} \ln \frac{y}{y^{*}} \\
& +\frac{p}{k}\left(1+\frac{b v^{*}}{1+a x^{*}}\right)\left(v-v^{*}-\int_{v^{*}}^{v} \frac{p y^{*}}{\frac{\beta x^{*} \tau}{1+a x^{*}+b \tau}} d \tau\right) .
\end{aligned}
$$

With Itô’s formula, we get

$$
\begin{aligned}
& d V_{21}(x, y, v)=L V_{21}(x, y, v) d t \\
& d V_{22}(x, y, v)=L V_{22}(x, y, v) d t \\
& d V_{23}(x, y, v)=L V_{23}(x, y, v) d t+\left[\frac{p y^{*}\left(1+a x+b v^{*}\right)}{\beta x v^{*}}-\frac{y^{*}}{y}\right] \frac{\sigma x v}{1+a x+b v} d B(t) .
\end{aligned}
$$

In detail,

$$
\begin{aligned}
L V_{21}(x, y, v)= & \left(x-x^{*}+y-y^{*}\right)(\lambda-q x-p y) \\
\leq & -q\left(x-x^{*}\right)^{2}-\frac{p}{2}\left(y-y^{*}\right)^{2}+\frac{(p+q)^{2}}{2 p}\left(x-x^{*}\right)^{2}, \\
L V_{22}(x, y, v)= & \left(v-v^{*}\right)(k y-u v) \\
\leq & -\frac{u}{2}\left(v-v^{*}\right)^{2}+\frac{k^{2}}{2 u}\left(y-y^{*}\right)^{2}, \\
L V_{23}(x, y, v)= & {\left[1-\frac{p y^{*}\left(1+a x+b v^{*}\right)}{\beta x v^{*}}\right]\left(\lambda-q x-\frac{\beta x v}{1+a x+b v}\right)+\left(1-\frac{y^{*}}{y}\right) } \\
& \cdot\left(\frac{\beta x v}{1+a x+b v}-p y\right)+\frac{p}{k}\left(1+\frac{b v^{*}}{1+a x^{*}}\right)\left[1-\frac{p y^{*}\left(1+a x^{*}+b v\right)}{\beta x^{*} v}\right] \\
& \cdot(k y-u v)+\frac{\sigma^{2} x^{2} v^{2}}{2(1+a x+b v)^{2}}\left(\frac{1+b v^{*}}{\beta v^{*} x^{* 2}}+\frac{y^{*}}{y^{2}}\right) \\
= & q x^{*}+p y^{*}-q x-p y^{*} \cdot \frac{v}{v^{*}}-\frac{x^{*}}{x} \cdot \frac{1+a x+b v^{*}}{1+a x^{*}+b v^{*}} \cdot q x^{*}-\frac{x^{*}}{x} \\
& \cdot \frac{1+a x+b v^{*}}{1+a x^{*}+b v^{*}} \cdot p y^{*}+\frac{1+a x+b v^{*}}{1+a x^{*}+b v^{*}} \cdot q x^{*}+\frac{v}{v^{*}} \cdot \frac{1+a x+b v^{*}}{1+a x+b v} \cdot p y^{*} \\
& -\frac{y^{*}}{y} \cdot\left(\frac{\beta x v}{1+a x+b v}-p y\right)-\frac{p v^{*}}{k v}(k y-u v)+\frac{\sigma^{2} x^{2} v^{2}}{2(1+a x+b v)^{2}} \\
& \cdot\left(\frac{1+b v^{*}}{\beta v^{*} x^{* 2}}+\frac{y^{*}}{y^{2}}\right) \\
\leq & -\frac{q\left(1+b v^{*}\right)}{x\left(1+a x^{*}+b v^{*}\right)}\left(x-x^{*}\right)^{2}-\frac{p y^{*} b(1+a x)}{v^{*}\left[1+(a+b) N_{m}\right]\left(1+a x+b v^{*}\right)} \\
& \cdot\left(v-v^{*}\right)^{2}+\frac{1}{2}(\sigma x v)^{2}\left(\frac{1+b v^{*}}{\beta v^{*} x^{* 2}}+\frac{y^{*}}{N_{\min }^{2}}\right)
\end{aligned}
$$




$$
\begin{aligned}
\leq & -\frac{q\left(1+b v^{*}\right)}{N_{m}\left[1+(a+b) N_{m}\right]}\left(x-x^{*}\right)^{2}-\frac{b p y^{*}}{v^{*}\left[1+(a+b) N_{m}\right]^{2}}\left(v-v^{*}\right)^{2} \\
& +\frac{1}{2}(\sigma x v)^{2}\left(\frac{1+b v^{*}}{\beta v^{*} x^{* 2}}+\frac{y^{*}}{N_{\min }^{2}}\right)
\end{aligned}
$$

where $N_{m}=\max \left\{N_{\max }, V_{\max }\right\}$.

Hence

$$
\begin{aligned}
& L V_{2}(x, y, v) \\
&=L V_{21}(x, y, v)+\frac{p u}{2 k^{2}} L V_{22}(x, y, v)+c_{1} L V_{23}(x, y, v) \\
& \leq- q\left(x-x^{*}\right)^{2}-\frac{p}{2}\left(y-y^{*}\right)^{2}+\frac{(p+q)^{2}}{2 p}\left(x-x^{*}\right)^{2} \\
& \quad+\frac{p u}{2 k^{2}}\left[-\frac{u}{2}\left(v-v^{*}\right)^{2}+\frac{k^{2}}{2 u}\left(y-y^{*}\right)^{2}\right]+c_{1}\left\{-\frac{q\left(1+b v^{*}\right)}{N_{m}\left[1+(a+b) N_{m}\right]}\right. \\
&\left.\cdot\left(x-x^{*}\right)^{2}-\frac{b p y^{*}}{v^{*}\left[1+(a+b) N_{m}\right]^{2}} \cdot\left(v-v^{*}\right)^{2}+\frac{1}{2}(\sigma x v)^{2}\left(\frac{1+b v^{*}}{\beta v^{*} x^{* 2}}+\frac{y^{*}}{N_{\min }^{2}}\right)\right\} .
\end{aligned}
$$

From the constant $c_{1}=\frac{N_{m}\left[1+(a+b) N_{m}\right](p+q)^{2}}{2 p q\left(1+b v^{*}\right)}$ in Theorem 4.1 and the inequality $a^{2} \leq 2(a-$ $\left.b^{*}\right)^{2}+2 b^{* 2}$, we get

$$
\begin{aligned}
& L V_{2}(x, y, v) \\
& \quad \leq-q\left(x-x^{*}\right)^{2}-\frac{p}{4}\left(y-y^{*}\right)^{2}-A\left(v-v^{*}\right)^{2}+\frac{B}{2}(\sigma x v)^{2} \\
& \quad \leq-q\left(x-x^{*}\right)^{2}-\frac{p}{4}\left(y-y^{*}\right)^{2}-A\left(v-v^{*}\right)^{2}+B N_{m} \sigma^{2}\left[\left(x-x^{*}\right)^{2}+x^{* 2}\right] \\
& \quad \leq-\left(q-B N_{m} \sigma^{2}\right)\left(x-x^{*}\right)^{2}-\frac{p}{4}\left(y-y^{*}\right)^{2}-A\left(v-v^{*}\right)^{2}+K,
\end{aligned}
$$

or

$$
\begin{aligned}
L V_{2}(x, y, v) \\
\quad \leq-q\left(x-x^{*}\right)^{2}-\frac{p}{4}\left(y-y^{*}\right)^{2}-A\left(v-v^{*}\right)^{2}+\frac{B}{2}(\sigma x v)^{2} \\
\quad \leq-q\left(x-x^{*}\right)^{2}-\frac{p}{4}\left(y-y^{*}\right)^{2}-A\left(v-v^{*}\right)^{2}+B N_{m} \sigma^{2}\left[\left(v-v^{*}\right)^{2}+v^{* 2}\right] \\
\quad \leq-q\left(x-x^{*}\right)^{2}-\frac{p}{4}\left(y-y^{*}\right)^{2}-\left(A-B N_{m} \sigma^{2}\right)\left(v-v^{*}\right)^{2}+K,
\end{aligned}
$$

where

$$
\begin{aligned}
& A=\frac{p u^{2}}{4 k^{2}}+\frac{c_{1} b p y^{*}}{v^{*}\left[1+(a+b) N_{m}\right]^{2}}, \\
& B=c_{1}\left(\frac{1+b v^{*}}{\beta v^{*} x^{* 2}}+\frac{y^{*}}{N_{\min }^{2}}\right), \\
& K=\max \left\{B N_{2}^{2} \sigma^{2} x^{* 2}, B N_{2}^{2} \sigma^{2} v^{* 2}\right\} .
\end{aligned}
$$


Wang et al. Advances in Difference Equations

(2020) 2020:493

Page 11 of 14

Hence

$$
\begin{aligned}
d V_{2}(x, y, v) \leq & \left\{-\left(q-B N_{m} \sigma^{2}\right)\left(x-x^{*}\right)^{2}-\frac{p}{4}\left(y-y^{*}\right)^{2}-A\left(v-v^{*}\right)^{2}+K\right\} d t \\
& +c_{1}\left[\frac{p y^{*}\left(1+a x+b v^{*}\right)}{\beta x v^{*}}-\frac{y^{*}}{y}\right] \frac{\sigma x v}{1+a x+b v} d B(t),
\end{aligned}
$$

or

$$
\begin{aligned}
d V_{2}(x, y, v) \leq & \left\{-q\left(x-x^{*}\right)^{2}-\frac{p}{4}\left(y-y^{*}\right)^{2}-\left(A-B N_{m} \sigma^{2}\right)\left(v-v^{*}\right)^{2}+K\right\} d t \\
& +c_{1}\left[\frac{p y^{*}\left(1+a x+b v^{*}\right)}{\beta x v^{*}}-\frac{y^{*}}{y}\right] \frac{\sigma x v}{1+a x+b v} d B(t) .
\end{aligned}
$$

Integrating both sides of (4.1) and (4.2) from 0 to $t$ and taking expectations, we obtain

$$
\begin{aligned}
0 \leq & E\left[V_{2}(x(t), y(t), v(t))\right] \\
\leq & E\left[V_{2}(x(0), y(0), v(0))\right] \\
& +E \int_{0}^{t}\left\{-\left(q-B N_{m} \sigma^{2}\right)\left(x-x^{*}\right)^{2}-\frac{p}{4}\left(y-y^{*}\right)^{2}-A\left(v-v^{*}\right)^{2}+K\right\} d r,
\end{aligned}
$$

or

$$
\begin{aligned}
0 \leq & E\left[V_{2}(x(t), y(t), v(t))\right] \\
\leq & E\left[V_{2}(x(0), y(0), v(0))\right] \\
& +E \int_{0}^{t}\left\{-q\left(x-x^{*}\right)^{2}-\frac{p}{4}\left(y-y^{*}\right)^{2}-\left(A-B N_{m} \sigma^{2}\right)\left(v-v^{*}\right)^{2}+K\right\} d r .
\end{aligned}
$$

This implies that

$$
\begin{aligned}
& E \int_{0}^{t}\left\{\left(q-B N_{m} \sigma^{2}\right)\left(x-x^{*}\right)^{2}+\frac{p}{4}\left(y-y^{*}\right)^{2}+A\left(v-v^{*}\right)^{2}\right\} d r \\
& \quad \leq E[V(x(0), y(0), v(0))]+K t
\end{aligned}
$$

or

$$
\begin{aligned}
& E \int_{0}^{t}\left\{q\left(x-x^{*}\right)^{2}+\frac{p}{4}\left(y-y^{*}\right)^{2}+\left(A-B N_{m} \sigma^{2}\right)\left(v-v^{*}\right)^{2}\right\} d r \\
& \quad \leq E[V(x(0), y(0), v(0))]+K t .
\end{aligned}
$$

Then

$$
\limsup _{t \rightarrow \infty} \frac{1}{t} E \int_{0}^{t}\left\{\left(q-B N_{m} \sigma^{2}\right)\left(x-x^{*}\right)^{2}+\frac{p}{4}\left(y-y^{*}\right)^{2}+A\left(v-v^{*}\right)^{2}\right\} d r \leq K,
$$

or

$$
\limsup _{t \rightarrow \infty} \frac{1}{t} E \int_{0}^{t}\left\{q\left(x-x^{*}\right)^{2}+\frac{p}{4}\left(y-y^{*}\right)^{2}+\left(A-B N_{m} \sigma^{2}\right)\left(v-v^{*}\right)^{2}\right\} d r \leq K
$$


By the conditions of the theorem, $\sigma^{2}<\min \left\{\frac{q}{N_{m}^{2} B}, \frac{A}{N_{m}^{2} B}\right\}$, and setting

$$
M=\min \left\{q-B N_{m} \sigma^{2}, A-B N_{m} \sigma^{2}, \frac{p}{4}\right\}
$$

we have

$$
\limsup _{t \rightarrow \infty} \frac{1}{t} E \int_{0}^{t}\left[\left(x(r)-x^{*}\right)^{2}+\left(y(r)-y^{*}\right)^{2}+\left(v(r)-v^{*}\right)^{2}\right] d r \leq \frac{K}{M}
$$

The theorem is proved.

\section{Numerical simulations}

To verify our analytical results, we conduct numerical simulations of the solution of model (1.2) using the Euler-Maruyama method [23]. Then the discretization equation of the model (1.2) is

$$
\left\{\begin{array}{l}
x_{n+1}=x_{n}+\left(\lambda-q x_{n}-\frac{\beta x_{n} v_{n}}{1+a x_{n}+b v_{n}}\right) \Delta t-\sigma \frac{x_{n} v_{n}}{1+a x_{n}+b v_{n}} \sqrt{\Delta t} \zeta \\
y_{n+1}=y_{n}+\left(\frac{\beta x_{n} v_{n}}{1+a x_{n}+b v_{n}}-p y_{n}\right) \Delta t+\sigma \frac{x_{n} v_{n}}{1+a x_{n}+b v_{n}} \zeta, \\
v_{n+1}=v_{n}+\left(k y_{n}-u_{n} v_{n}\right) \Delta t
\end{array}\right.
$$

where $\Delta t>0$, and $\zeta$ consists of $N(0,1)$-distributed independent random variables.

In Theorem 3.2, we take the initial value $(6,9,14)$ and parameters $\lambda=4.8, \beta=0.11$, $k=0.8, q=0.3, p=0.4, u=0.5, a=0.4, b=0.3, \Delta t=10^{-2}$, and we compute that $R_{0} \approx$ $0.9514<1$ and the uninfected steady state $E_{0}=(16,0,0)$. The intensity of white noise is relevant to the value of $\sigma$. In the simulation, we take different values $\sigma: 0.06$ and 0.1 , which conform to the conditions in Theorem 3.2. Figure 1 shows that the solution of the model (1.2) is stochastically stable under certain conditions, which is consistent with the conclusion of Theorem 3.2. The fluctuations reduce as the noise intensity $\sigma^{2}$ decreases before the solution up to the uninfected steady state.

Let $\beta=0.13$, and the other parameters be the same as above, then $R_{0} \approx 1.1243>1$, and the infected steady state $E^{*} \approx(14.0833,1.4375,2.3)$. Let $\sigma=0.02$ and $\sigma=0.06$, respectively, satisfying conditions of Theorem 4.1. In Fig. 2, the solution of the model (1.2) fluctuates randomly around $E^{*} \approx(14.0833,1.4375,2.3)$ of the model $(1.1)$. When $\sigma^{2}$ is smaller, the fluctuation intensity is smaller and closer to $E^{*}$. However, when $\sigma=0$, the conclusions of models (1.2) and (1.1) are the same.

\section{Conclusion}

We proposed a new stochastic HIV model with a Beddington-DeAngelis functional response in our work, and discussed unique positivity globally and asymptotic behavior of the solution in the model by constructing various types of Lyapunov functions. More precisely, when the basic reproductive number is smaller than or equal to 1 , the asymptotic behavior of uninfected steady state is stochastically stable. When the basic reproductive number is higher than 1, it oscillates randomly around the infected steady state in the corresponding deterministic HIV model. In the numerical simulations, we take different noise intensities under certain conditions and the transmission coefficients but keep the other parameters unchanged to be verify our results. Moreover, when the noise intensity 

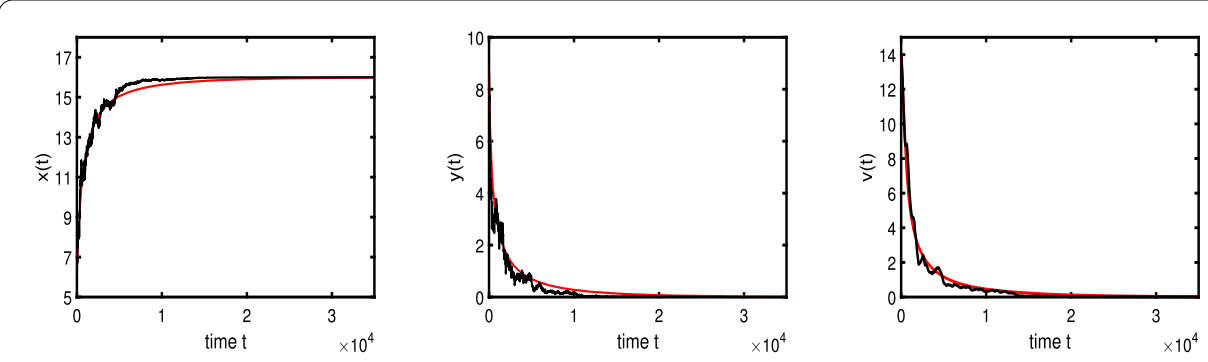

(a) $\sigma=0.06$
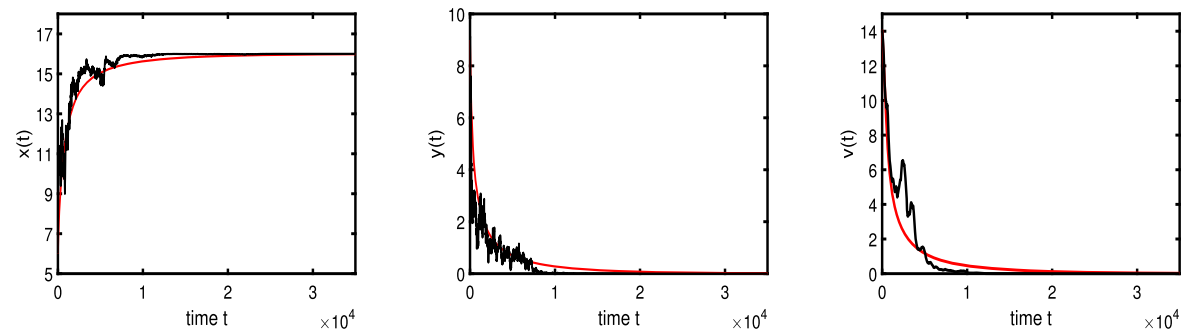

(b) $\sigma=0.1$

Figure 1 Given $R_{0}<1$, the relationship between trajectories of deterministic model (1.1) and stochastic model (1.2)
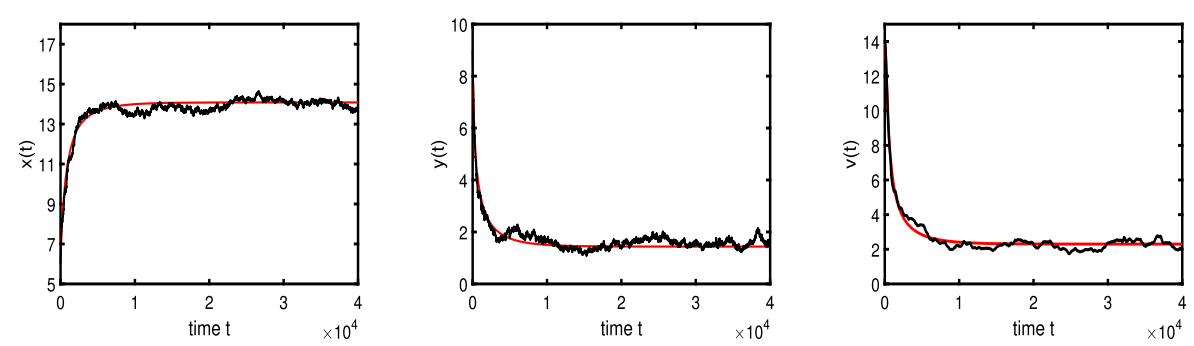

(a) $\sigma=0.02$
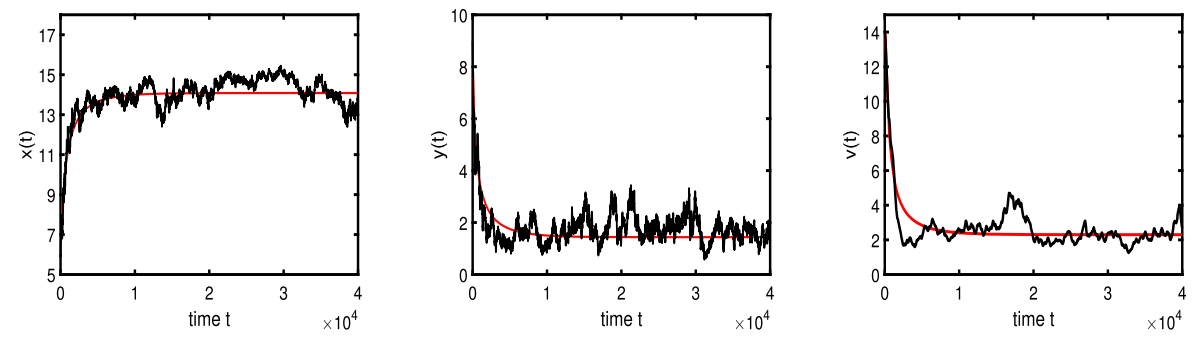

(b) $\sigma=0.06$

Figure 2 Given $R_{0}>1$, the relationship between trajectories of deterministic model (1.1) and stochastic model (1.2)

decreases, oscillations around the corresponding deterministic model reduce. Our model can well reflect the mutual interference between the concentration of uninfected $\mathrm{CD} 4^{+} \mathrm{T}$ cells and HIV viruses biologically. The Beddington-DeAngelis functional response of the 
infection rate of HIV with additional noise conforms to the randomness in real systems. These results can extend HIV models but the threshold of the noise intensity was rather small, which is improved in this paper.

\section{Acknowledgements}

The authors would like to thank the associate editor and the reviewers for their careful reading and insightful suggestions, which have helped improve this paper.

\section{Funding}

The work was supported by the National Key Research and Development Program of China (No. 2016YFC1401800)

\section{Availability of data and materials}

Data sharing not applicable to this article as no datasets were generated or analyzed during the current study.

\section{Competing interests}

The authors declare that they have no competing interests.

\section{Authors' contributions}

All authors contributed equally and significantly in writing this paper. All authors have read and approved the final paper.

\section{Author details}

${ }^{1}$ College of Computer Meteorology and Oceanography, National University of Defense Technology, Changsha, China.

${ }^{2}$ College of Computer Science and Technology, National University of Defense Technology, Changsha, China.

\section{Publisher's Note}

Springer Nature remains neutral with regard to jurisdictional claims in published maps and institutional affiliations.

Received: 25 October 2019 Accepted: 17 August 2020 Published online: 15 September 2020

\section{References}

1. Hernandez-Vargas, E.A., Middleton, R.H.: Modelling the three stages in HIV infection. J. Theor. Biol. 320, 33-40 (2013)

2. Nowak, M.A., Bangham, C.R.M.: Population dynamics of immune responses to persistent virus. Science $272,74-79$ (1996)

3. Huang, G., Ma, W.B., Takeuchi, Y.: Global properties for virus dynamics model with Beddington-DeAngelis functional response. Appl. Math. Lett. 22, 1690-1693 (2009)

4. Beddington, J.R.: Mutual interference between parasites or predators and its effect on searching efficiency. J. Anim. Ecol. 44, 331-340 (1975)

5. DeAngelis, D.L., Goldstein, R.A., O'Neill, R.V.: A model for tropic interaction. Ecology 56, 881-892 (1975)

6. Dalal, N., Greenhalgh, D., Mao, X.R.: A stochastic model for internal HIV dynamics. J. Math. Anal. Appl. 341, 1084-1101 (2008)

7. Wang, S.X., Dong, L.Z., Wang, X.Y.: Asymptotic behaviors of the solutions for a stochastic SIQR system with vaccination. In: 2016 3rd International Conference on Systems and Informatics (ICSAI), pp. 620-626 (2016). https://doi.org/10.1109/ICSAI.2016.7811028

8. Li, D., Ma, W.B.: Asymptotic properties of an HIV-1 infection model with time delay. J. Math. Anal. Appl. 335, 683-691 (2007)

9. Song, X.Y., Neumann, A.U.: Global stability and periodic solution of the viral dynamics. J. Math. Anal. Appl. 329 281-297 (2007)

10. Yan, C., Wang, W.D.: Modeling HIV dynamics under combination therapy with inducers and antibodies. Bull. Math. Biol. 81, 2625-2648 (2019)

11. Tuckwell, H.C., Le Corfec, E.: A stochastic model for early HIV-1 population dynamics. J. Theor. Biol. 195, 451-463 (1998)

12. Kouyos, R.D., Althaus, C.L., Bonhoeffer, S.: Stochastic or deterministic: what is the effective population size of HIV-1? Trends Microbiol. 14, 507-511 (2016)

13. Nijhuis, M., Boucher, C., Schipper, P.: Stochastic processes strongly influence HIV-1 evolution during suboptimal protease-inhibitor therapy. Proc. Natl. Acad. Sci. USA 95, 14441-14446 (1998)

14. Feng, T., Qiu, Z.P., Meng, X.Z.: Dynamics of a stochastic hepatitis c virus system with host immunity. Discrete Contin. Dyn. Syst., Ser. B 24, 6367-6385 (2019)

15. Feng, T., Qiu, Z.P., Meng, X.Z:: Analysis of a stochastic recovery-relapse epidemic model with periodic parameters and media coverage. J. Appl. Anal. Comput. 9, 1007-1021 (2019)

16. Mao, X.R.: Stochastic Differential Equations and Applications. Elsevier, Amsterdam (2007)

17. Wang, Y., Jiang, D.Q., Hayat, T.: Stationary distribution of an HIV model with general nonlinear incidence rate and stochastic perturbations. J. Franklin Inst. 356, 6610-6637 (2019)

18. Liu, X.Q., Zhong, S.M., Xiang, L.J.: Asymptotic properties of a stochastic predator-prey model with Beddington-DeAngelis functional response. Int. J. Math. Comput. Sci. 8, 227-229 (2014)

19. Geiß, C., Manthey, R.: Comparison theorems for stochastic differential equations in finite and infinite dimensions. Stoch. Process. Appl. 53, 23-35 (1994)

20. Friedman, A.: Stochastic Difference Equations and Their Applications. Academic Press, New York (1976)

21. Arnold, L.: Stochastial Difference Equations: Theory and Applications. Wiley, New York (1972)

22. McNabb, A.: Comparison theorems for differential equations. J. Math. Anal. Appl. 119, 417-428 (1986)

23. Higham, D.J.: An algorithmic introduction to numerical simulation of stochastic differential equations. SIAM Rev. 43 525-546 (2001) 